\title{
Stellar activity and magnetism studied by optical interferometry
}

\author{
K. Rousselet-Perraut ${ }^{1}$, C. Stehlé ${ }^{2}$, T. Lanz ${ }^{2,3,4}$, J. B. Le Bouquin ${ }^{1}$, T. Boudoyen ${ }^{1}$, \\ M. Kilbinger ${ }^{5}$, O. Kochukhov ${ }^{6}$, and S. Jankov ${ }^{7}$ \\ ${ }^{1}$ Laboratoire d'Astrophysique de l'Observatoire de Grenoble, BP 53, 38041 Grenoble Cedex 9, France \\ 2 Laboratoire de l'Univers et de ses THéories, UMR 8102, Observatoire de Paris, 5 place Jules Janssen, 92195 Meudon, France \\ 3 Department of Astronomy, University of Maryland, College Park, MD 20742, USA \\ ${ }^{4}$ NASA Goddard Space Flight Center, Code 681, Greenbelt, MD 20771, USA \\ 5 Institut für Astrophysik und Extraterrestrische Forschung, Universität Bonn, Auf dem Hügel 71, 53121 Bonn, Germany \\ ${ }^{6}$ Uppsala Astronomical Observatory, Box 515, 751 20, Uppsala, Sweden \\ 7 Observatoire de la Côte d'Azur, Département Fresnel, CNRS UMR 6528, 06460 Saint Vallier de Thiey, France
}

\begin{abstract}
By means of numerical simulations, we investigate the ability of optical interferometry, via the fringe phase observable, to address stellar activity and magnetism. To derive abundance maps and stellar rotation axes, we use color differential interferometry which couples high angular resolution to high spectral resolution. To constrain magnetic field topologies, we add to this spectro-interferometer a polarimetric mode. Two cases of well-known Chemically Peculiar (CP) stars $\left(\beta \mathrm{CrB}\right.$ and $\left.\alpha^{2} \mathrm{CVn}\right)$ are simulated to derive instrumental requirements to obtain 2D-maps of abundance inhomogeneities and magnetic fields. We conclude that the near-infrared instrument AMBER of the VLTI will allow us to locate abundance inhomogeneities of CP stars larger than a fraction of milliarcsecond whereas the polarimetric mode of the French GI2T/REGAIN interferometer would permit one to disentangle various magnetic field topologies on CP stars. We emphasize the crucial need for developing and validating inversion algorithms so that future instruments on optical aperture synthesis arrays can be optimally used.
\end{abstract}

Key words. techniques: interferometric - techniques: polarimetric - stars: chemically peculiar - stars: magnetic fields stars: activity

\section{Introduction}

Stellar magnetism and activity have been most often studied with various spectro-polarimetric techniques, such as the analysis of the shape of line profiles in different Stokes parameters (the moment technique proposed by Mathys (1988)), the Broad Band Linear Polarization technique (Leroy et al. 1993), the Zeeman-Doppler (Semel 1989; Donati 1996) or the MagneticDoppler (Piskunov \& Kochukhov 2002) Imaging techniques (so-called ZDI and MDI techniques respectively). Generally, however, the poor angular resolution drastically limits detection and/or diagnosis since the polarized signals are averaged over the stellar surface and thus cancel because of symmetry. This is why optical interferometry appears to be very attractive to address stellar activity by providing an high angular resolution (Wittkowski et al. (2002) and references herein). In this astrophysical context, spectrometric information is also of strong interest: the spectrograph of the GI2T/REGAIN Interferometer (Mourard et al. 2002) has allowed one to constrain the radiative wind of hot stars (Vakili et al. 1997; Bério et al. 1999) or oscillations in Be stars (Vakili et al. 1998), and the high spectral

Send offprint requests to: K. Rousselet-Perraut, e-mail: karine.perraut@obs.ujf-grenoble.fr mode of AMBER (Petrov et al. 2000) is required to constrain complex patchy stellar surfaces and/or environments. Finally polarimetric interferometry allows one to resolve local polarized stellar features and is thus a powerful tool for studying scattering phenomena, magnetism, etc.: the first attempts of interfero-polarimetric observations have been reported essentially for studies of scattering and mass-loss phenomena inside hot and extended environments (Hanbury Brown et al. 1974; Vakili 1981; Rousselet-Perraut et al. 1997).

Depending on the angular resolution achieved, optical interferometry can be a tool for studying various classes of objects. As an example, the 1 milli-arcsec (1 mas) obtained with the European VLTI array (Glindemann et al. 2003) is of strong interest within the context of stellar physics, e.g. to partially resolve Young Stellar Objects and thus provide new insights into stellar formation and star forming regions. Further in the future, the fiber network OHANA linking the Hawaiian telescopes on Mauna Kea (Perrin et al. 2003) will provide angular resolutions of a fraction of a mas in the near-infrared range, which will be of benefit to many fields of investigation of stellar physics (e.g. star forming regions, giant stars, main sequence stars, etc.). 
In Chesneau et al. (2000) we proposed to investigate the ability of spectro-(polari)metric interferometry to constrain:

- abundance inhomogeneities and stellar rotation axes by spectro-interferometry (also called color differential interferometry);

- scattering and mass-loss phenomena by polarimetric interferometry;

- magnetism by spectro-polarimetric interferometry (also called SPIN).

The first step of our investigation is reported by Rousselet-Perraut et al. (2000) and consists of a numerical experiment for studying magnetic field topology of Ap stars by neglecting the effects of radiative transfer, stellar rotation, etc. We concluded from this crude approach that SPIN could be a powerful tool for studying stellar activity and magnetism and that a more robust and detailed modelling was required in order to reliably prepare, and later interpret, future observations made with optical aperture synthesis arrays. The present paper deals with this detailed modelling and simulates two cases on two existing interferometers. We first describe the fringe phase observable (Sect. 2) and illustrate its usefulness to determine stellar rotation axes, to detect stellar spots or to study magnetic field topologies (Sect. 3). Spot detection and magnetic topology study applications are illustrated in detail for two well-known Chemically Peculiar (CP) stars, $\beta \mathrm{CrB}$ and $\alpha^{2} \mathrm{CVn}$, assumed to be observed either with the GI2T/REGAIN interferometer or with AMBER on the VLTI (Sect. 4). The signal amplitudes and accuracies are discussed within the context of current and planned instruments.

\section{The differential fringe phase}

\subsection{Observable}

A two-telescope interferometer can sample the Fourier transform $|\widetilde{O}| \mathrm{e}^{i \psi}$ of a stellar brightness distribution $\mathrm{O}(\boldsymbol{\alpha})$ at spatial frequency $\boldsymbol{B} / \lambda$ where $\boldsymbol{B}$ stands for the interferometric baseline vector projected on the sky and $\lambda$ is the mean wavelength at which the interferometer operates.

Fringe phase information is provided by the position of the brightest fringe of the interferogram but, in practice, is corrupted by the Earth atmosphere. Several techniques such as phase referencing (Colavita 1992) or closure phase (Jennison 1958) allow one to retrieve phase information. It has been also demonstrated (Petrov 1988) that differential phases between two wavelengths, $\lambda_{1}$ and $\lambda_{2}$, can be measured by color differential interferometry, provided that the object is partially resolved and a spectrometer is coupled to the interferometer. In this case, we record phase measurements for each spectral channel. The differential fringe phase between two spectral channels remains mostly unbiased by atmospheric contributions. Such a technique can be generalized to polarimetric measurements: differential phases between different spectral channels can be measured for the four Stokes parameters, provided that a spectro-polarimeter is coupled to the interferometer (Fig. 1).

\subsection{Link to the stellar parameters}

From the Van-Cittert Zernike theorem, the fringe phase is given by:

$\psi \sim \frac{\int \sin (2 \pi \boldsymbol{\alpha} \boldsymbol{B} / \lambda) \cdot O(\boldsymbol{\alpha}) \mathrm{d}^{2} \alpha}{\int \cos (2 \pi \boldsymbol{\alpha} \boldsymbol{B} / \lambda) \cdot O(\boldsymbol{\alpha}) \mathrm{d}^{2} \alpha}$

with $O(\alpha)$, the object intensity distribution.

If the object is unresolved or marginally resolved, then $2 \pi \boldsymbol{\alpha} \boldsymbol{B} / \lambda \ll 1$ and the fringe phase expression becomes:

$\psi \sim 2 \pi \frac{\int \boldsymbol{\alpha} . O(\boldsymbol{\alpha}) \mathrm{d}^{2} \alpha}{\int O(\boldsymbol{\alpha}) \mathrm{d}^{2} \alpha} \cdot \frac{\boldsymbol{B}}{\lambda} \sim \boldsymbol{p} .2 \pi \frac{\boldsymbol{B}}{\lambda}$

with $\boldsymbol{p}$, the stellar photocenter:

$\boldsymbol{p}=\frac{\int \boldsymbol{\alpha} \cdot O(\boldsymbol{\alpha}) \mathrm{d}^{2} \alpha}{\int O(\boldsymbol{\alpha}) \mathrm{d}^{2} \alpha}$.

The fringe phase is thus proportional to the photocenter of the intensity distribution and is equivalent to the first moment of the intensity distribution (see Jankov et al. (2001) for the whole formalism).

\subsection{Calibration}

In practice, interferometric signals are affected by instrumental polarization due to the interferometer itself. The optics of one interferometric arm can introduce (Rousselet-Perraut et al. 1996):

- an intensity attenuation which can differ for each polarization component (i.e. natural, $s$ and $p$ linearly polarized components, and left and right circularly polarized components);

- a phase shift between the reference polarization directions: a cross-talk effect appears between the different polarized components;

- a rotation of the reference system of the polarization directions.

All these effects are well known in (spectro-)polarimetry and spectro-polarimeters are designed to minimize them. In optical interferometry, the fringe phase is only affected by the differential phase shift, which introduces an offset in the fringe position. Such an instrumental effect can be easily substracted, either by observing a calibrator of the same spectral type (such a calibrator can be an unpolarized as well as unresolved star), or by computing differential fringe phases between close spectral channels. For instance, if we measure phase signals in emission or absorption lines, the instrumental effect can be considered as achromatic in such a narrow spectral range. The differential fringe phase throughout the line profile is without bias. Within the context of polarimetric measurements, another calibration technique consists of intercorrelating the two polarized interferograms, right and left ones or $s$ and $p$ ones (Chesneau et al. 2001). 


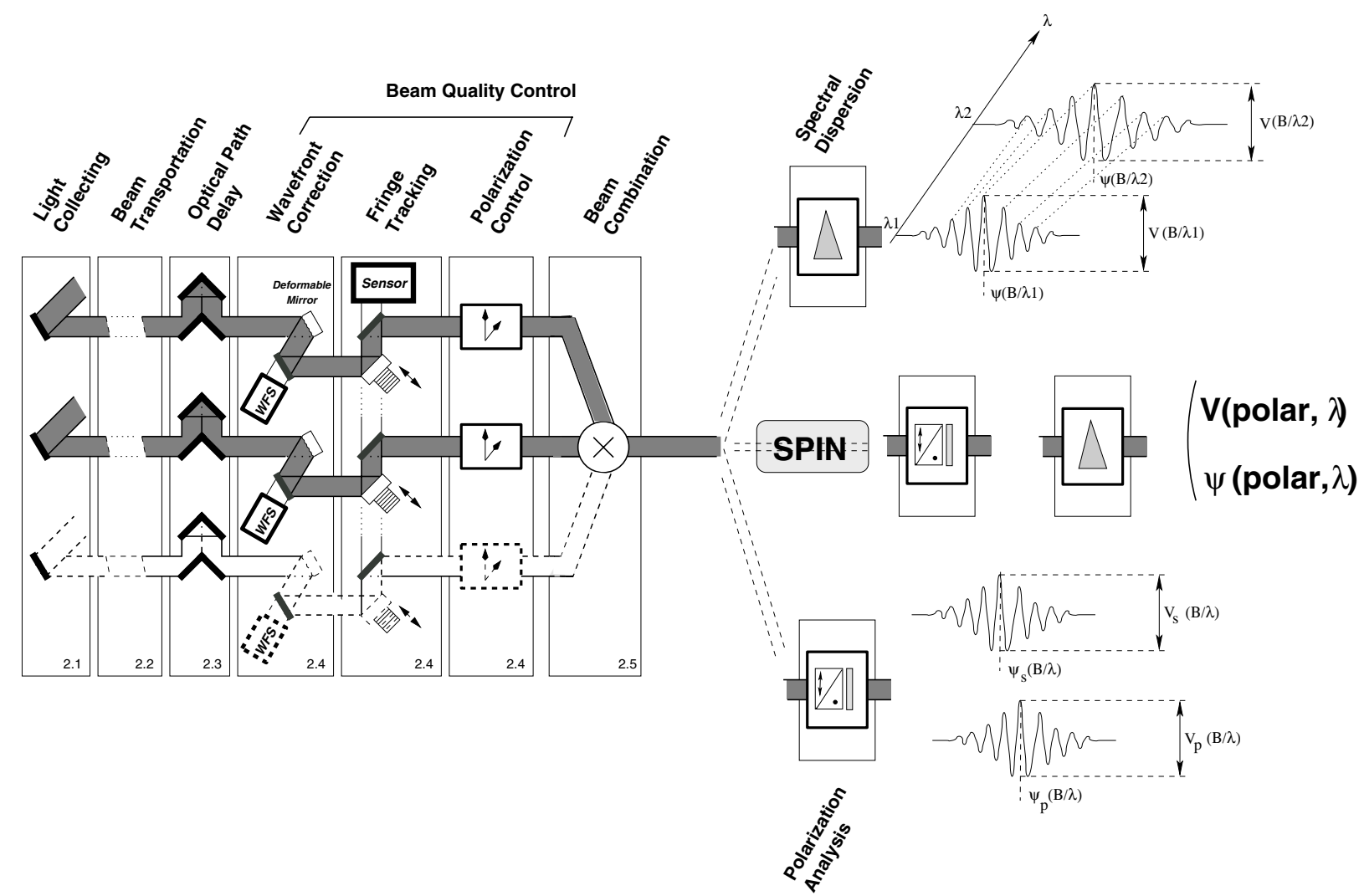

Fig. 1. An interferometer (left) combines two or more beams (a third beam is displayed at the bottom with dashed lines) and produces interference fringes whose visibility $V$ and fringe phase $\psi$ (i.e. position of the brightest fringe) are recorded. In "classical" interferometry, visibility and phase are recorded across a spectral band. If the interferometer is complemented with a spectrograph (top), one can record visibilities and fringe phases in various spectral channels. Similarly, if the interferometer is equipped with a polarimeter (bottom), one records visibilities and fringe phases in various polarization directions (as an example, we display the recording of visibility and phase for both linearly polarized directions $s$ and $p$ ). The Spectro-Polarimetric INterferometry technique (SPIN) couples an interferometer with a spectro-polarimeter (middle) and allows one to record visibilities and fringe phases in various polarization directions for each spectral channel.

\section{Stellar activity and magnetism studied by optical interferometry}

In the following, we consider two kinds of fringe phase measurements in the partially resolved range:

- the fringe phase $\psi_{I}$ in the $I$-Stokes parameter (i.e. in natural light) obtained with a spectro-interferometer. The differential phases are computed between the continuum and the spectral line under study;

- the fringe phase $\psi_{V}$ in the $V$-Stokes parameter (i.e. in circularly polarized light) obtained with a spectro-polarimetric interferometer (which measures the fringe positions in the right and left polarization directions). The differential phases are computed between the continuum and the spectral line under study. These measurements concern the magnetism study via the Zeeman effect analysis.

In this section we only illustrate the fringe phase usefulness to probe stellar activity and magnetism. Detailed science cases and instrumental requirements are given in the following sections within the context of existing or planned interferometers.

- Rotation axis determination: during the stellar rotation, the interferometer "sees" across spectral Doppler profile iso-velocity strips of the star. The photocenter moves across the spectral line. This displacement obviously varies with the rotational velocity and also depends on the interferometric baseline orientation with respect to the stellar rotation axis. Measuring fringe phases $\psi_{I}$ across a Doppler profile during Earth-rotation synthesis is a means for determining the stellar rotator orientation on the sky (Lagarde et al. 1995). This orientation will significantly help existing (Zeeman-)Doppler Imaging inversion methods.

- Spot detection: differential fringe phase $\psi_{I}$ can used to locate spots on stellar surfaces (Jankov et al. 2003). Since it is equivalent to the first moment of the intensity distribution, this observable has a large sensitivity in the limb regions (Fig. 2): a spot at the stellar limb (which is the case of the Southern spot) has a weak spectral signature, even for a favorable line of sight inclined by $50^{\circ}$ (a). On the other hand, such a spot generates a large photocenter (i.e. phase) signature during the stellar rotation, as illustrated by the photocenter signature in the $Y$ direction (c) when the spot is at the top, or by the photocenter signature in the $X$ direction (b) when the spot is on the right or on the left.

A further interesting property of the fringe phase is to provide access to absolute orientations via the phase sign, even for edge-on or pole-on geometries. While such stellar geometries cannot be reconstructed from spectral signatures due to the "mirror-effect" (Fig. 3a), the photocenter 


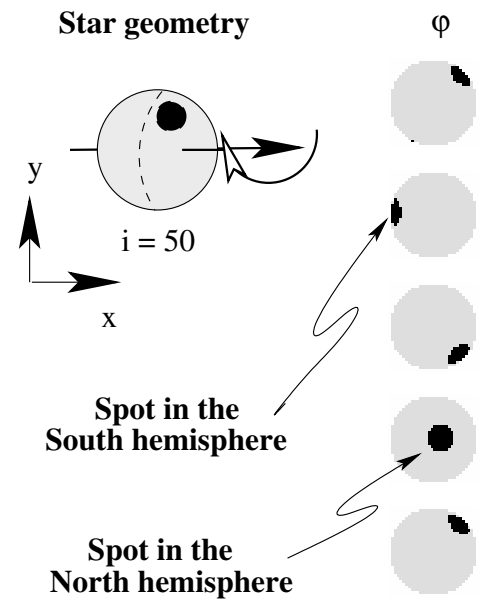

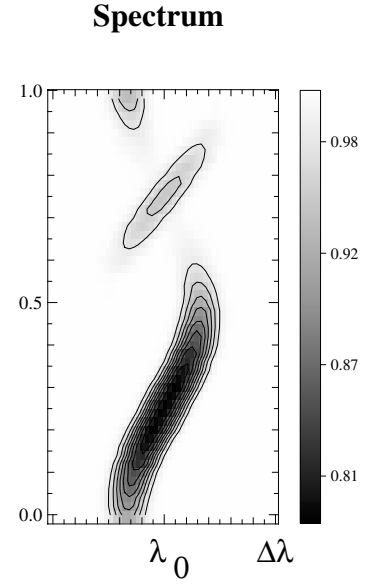

(a)

Photocenters

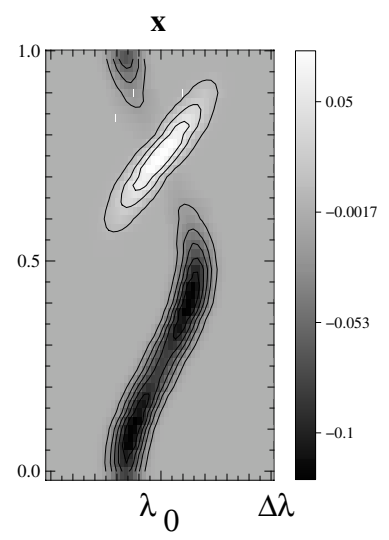

(b)

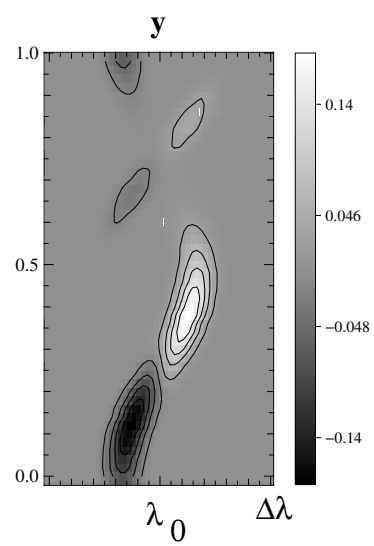

(c)

Fig. 2. Simulation of a patchy surface seen with a line of sight inclined by $50^{\circ}$ (left). Two chromium spots, one in each hemisphere, lead to a dynamical spectrum a) and to photocenter (i.e. fringe phase) signatures (b), c)) throughout the stellar rotation. The scale to the right of each graph corresponds either to the intensity in the line a), or to the fringe phase in radians (b), c)). The two spots have the same characteristics: the radius equals $18^{\circ}$; the abundance variation between the stellar surface and the spot equals 3 dex. The stellar diameter equals 0.88 mas, the rotational speed is $v \sin i=15 \mathrm{~km} \mathrm{~s}^{-1}$, the effective temperature is $T_{\text {eff }}=11500 \mathrm{~K}$, and $\log g=4 . \lambda_{0}=4824.12 \AA$ and the spectra extend over $\pm 0.47 \AA$ from the line center. The interferometric baseline equals $50 \mathrm{~m}$.
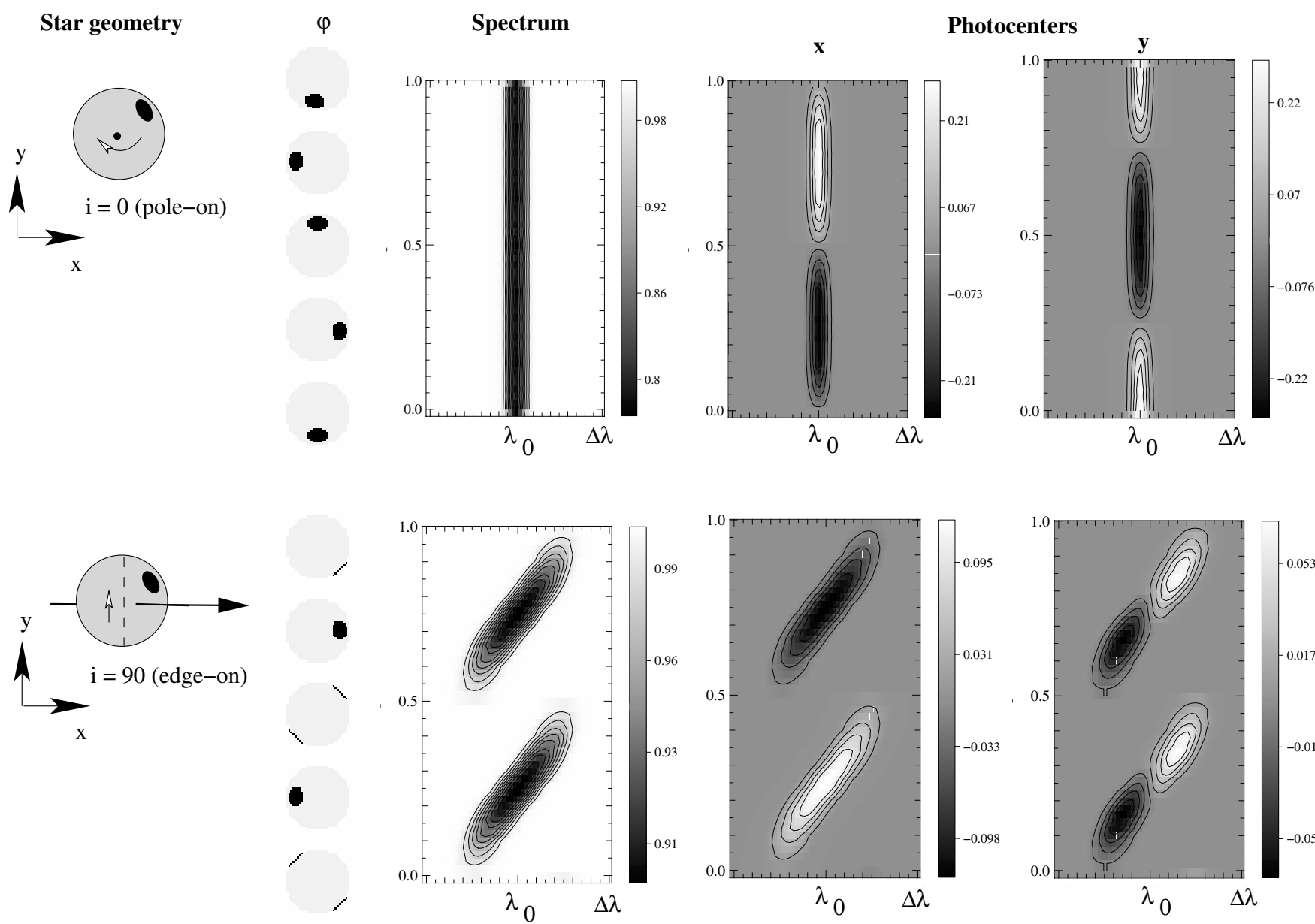

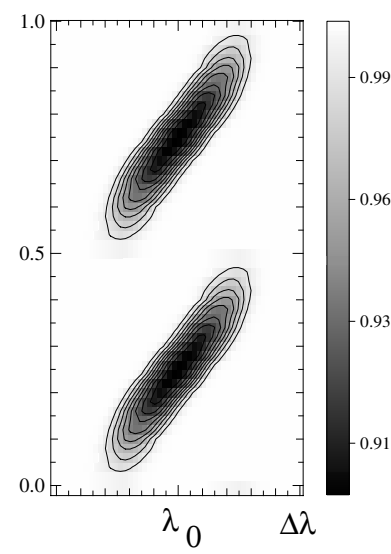

(a)

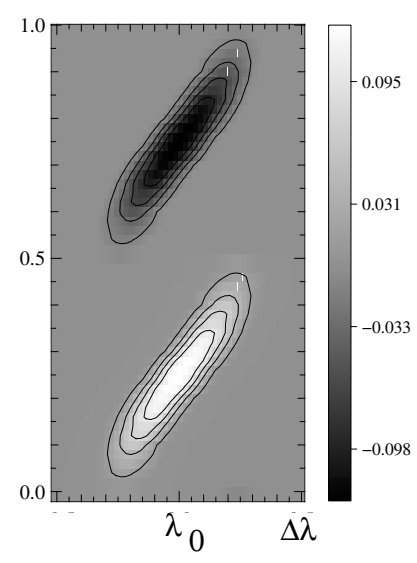

(b)

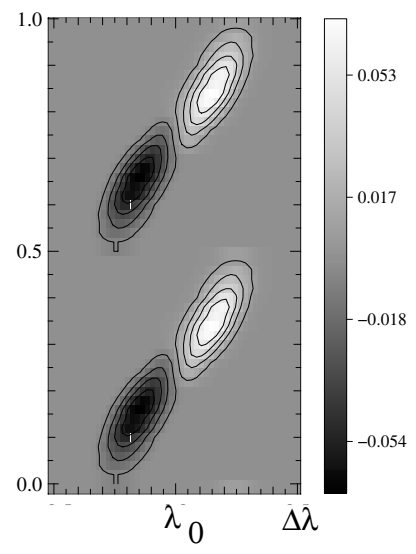

(c)

Fig. 3. Simulations of a pole-on rotator (top) and a edge-on rotator (bottom) with the same patchy surface and the same stellar and interferometric parameters as Fig. 2. Corresponding spectra a) and photocenter (i.e. fringe phase) signatures (b), c)) throughout the stellar rotation. 
signatures (i.e. the amplitude and sign of the fringe phase) along the $X$ and $Y$ directions $(\mathrm{b}, \mathrm{c})$ allow the degeneracy to be lifted and therefore the spots can be located.

- Mass-loss: polarimetric interferometry is a powerful tool for constraining stellar wind parameters of hot stars, especially in the case of dense winds or extended atmospheres (Chesneau \& Wolf 2003). This is therefore an attractive means for studying mass-loss phenomena.

- Magnetic field topology: a pedagogical example of a dipolar magnetic field seen from the equator illustrates the SPIN ability to study magnetic field topologies (various magnetic configurations are described and studied in Rousselet-Perraut et al. 2000). The rotation axis as well as the dipole axis is horizontal. The $V$-Stokes spectral profile (i.e., in circularly polarized light) has an " $S$ " shape (Fig. 4-bottom) well known in spectro-polarimetry and linked to the Zeeman splitting of the magnetically sensitive line. The stellar intensity maps in the $V$-Stokes parameter significantly vary across the spectral line (Fig. 4-top): these maps contain Doppler rotation information (we clearly show the iso-velocity strips) mixed with Zeeman splitting information (inversion of the magnetic field sign across the line). As a consequence, photocenter displacement can be observed throughout the line, which can be detected by spectro-polarimetric interferometry as a differential fringe phase $\psi_{V}$ inversion.

\section{Simulations of chemically peculiar stars observed with the existing interferometers}

\subsection{Sources}

We focus on Chemically Peculiar A and B stars (CP stars) since they exhibit strong chemical abundance inhomogeneities of one or more chemical elements, such as helium, silicon, chromium, strontium, or europium, and a large-scale organization of their magnetic field that produces a typical signature in circularly-polarized spectra. CP stars represent a major class of the known magnetic stars in the solar neighborhood and constitute ideal targets for studying how magnetic fields affect other physical processes occuring in stellar atmospheres. A significant gain in angular resolution will allow one to map abundance distributions and magnetic fields, which is an important key in addressing the fundamental question of the origin of the magnetic field in CP stars: both the fossil and the coredynamo theories have difficulty in explaining all the observed magnetic characteristics of CP stars (Moss 2001). Secondly, the magnetic field and the abundance inhomogeneities are so closely related that maps have to be obtained simultaneously to understand well the key role of magnetism in atmosphere structuration (Leblanc et al. 1994), in ion migration across the stellar surface (Michaud 1970), and in chemical stratification (Ryabchikova et al. 2002). However, very few abundance maps of CP stars are available today (e.g. Kochukhov et al. 2002 for $\alpha^{2} \mathrm{CVn}$ ) and very few maps of magnetic fields have been reconstructed via Zeeman-Doppler Imaging (e.g. Kochukhov et al. 2002 for $\alpha^{2} \mathrm{CVn}$ ) or by inversion of spectro-polarimetric data (Bagnulo et al. 2000 for $\beta \mathrm{CrB}$ ). Moreover, such inversion

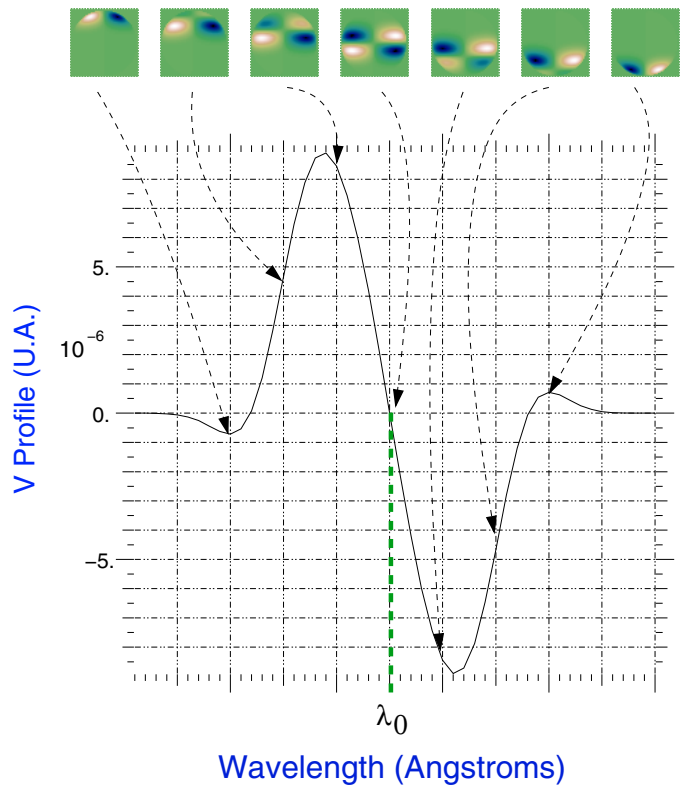

Fig. 4. Simulations in an iron line $\left(\lambda_{0}=6000 \AA\right)$ of a dipolar magnetic field $\left(g B_{\mathrm{d}}=4000 \mathrm{G}\right.$ with $g$ the Landé factor) seen from the equator with a rotational velocity of $v \sin i$ of $50 \mathrm{~km} \mathrm{~s}^{-1}$ and a spectral resolution of 30000 . The spectra extend over $\pm 0.2 \AA$ from the line center. The rotation axis as well as the dipole axis is horizontal. The intensity maps in the $V$-Stokes parameter (top) are displayed across the classical S-shape spectral profile in the $V$-Stokes parameter (bottom).

methods often lead to several magnetic field models that cannot be disentangled by classical spectro-polarimetric techniques. In this context, the fringe phase observable is a very attractive way to derive $2 \mathrm{D}$ abundance and magnetic maps.

\subsection{Numerical codes}

We have developed two numerical codes for $\mathrm{CP}$ stars to predict intensity maps at various stellar rotational phases and then derive fringe phases by Fourier Transformation at these rotational phases for various instrumental configurations.

- The first program, modpol, allows us to compute the fringe phase signals in the two Stokes components, I (natural light) and $V$ (circularly polarized light). It assumes that the emergent local profile has a Gaussian shape with a thermal broadening corresponding to the effective temperature $T_{\text {eff }}$, and a microturbulent broadening (although we have in general assumed a zero microturbulence). The other parameters are the oscillator strength and the local density of absorbing atoms, which is proportional to the line equivalent width, $W_{\lambda}$, for weak lines. $W_{\lambda}$ is calculated using the spectrum synthesis code SYNSPEC of Hubeny \& Lanz (2000). The effect of the magnetic field is included by a triplet Zeeman pattern characterized by the effective Landé factor $g_{\text {eff }}$. The limb-darkening effect is included through a linear law in $a_{0}(1-\cos \theta), \theta$ being the angle between the normal at the surface and the observer's line of sight. These parameters, the effective temperature, the Landé factor, the oscillator strength, the equivalent width, the local rotational velocity and also the magnetic field value allow the 
computation of the local $I$ and $V$ profiles. Thus modpol is very fast and user-friendly, but does not solve the radiative transfer equation. In particular, modpol does not take into account the profile variations due to saturation or complex Zeeman patterns.

- The second code, prloc, produces local emergent profiles in the $I$-Stokes component only, solving the LTE radiative transfer equation. It assumes a LTE plane-parallel atmospheric structure, calculated with ATLAS9 (Kurucz 1993). prloc solves the radiative transfer equation using the Feautrier method, following the Rybicki \& Hummer (1991) improved scheme. LTE populations and opacities (continuum opacities and line profiles) are computed following Synspec (Hubeny \& Lanz 2000). The relevant subroutines have been extracted from this code and are called by prloc. prloc generates emergent line profiles, taking into account the local chemical abundance and the Zeeman line splitting due to the local magnetic field. The anomalous Zeeman pattern is produced from Landé factors and quantum numbers of the upper and lower levels of the transition, assuming $L S$-rules. For each chemical species, the local abundance is parametrized and can be either derived from observations, or assumed by the user. From abundance and magnetic maps provided by Kochukhov et al. (2002), we have calculated integrated spectra of various metallic lines vs. the stellar rotational phase. We have then checked that the simulated spectra are in agreement with the observed ones (Fig. 5).

Such an unpolarized radiative transfer code is sufficient for the line computation of the spectrum in intensity (i.e. in the $I$-Stokes parameter). However, polarized radiative transfer has to be implemented to model observations in the $V$-Stokes parameter and to predict circularly polarized emergent line profiles for magnetic field detection. This upgrade is in progress.

Modelling of the hydrogen lines formed in a magnetic field is another interesting issue. We plan to derive 2D magnetic maps by measuring fringe phase in the $V$-Stokes parameter in the blue and red wings of hydrogen lines with a technique analogous to the Landstreet's approach in classical spectropolarimetry (Landstreet 1980). To this purpose, we can couple our current code with the code of polarized hydrogen lines applied to $\mathrm{H} \beta$ line by Brillant et al. (1998) and Mathys et al. (2000) and also to Lyman $\alpha$ line by Stehlé et al. (2000). The latter code allows us to compute the various hydrogen lines in the different Stokes parameters. It has been successfully used to revisit the interpretation of photopolarimetric observations of Balmer lines in terms of mean longitudinal magnetic field. Another possibility would be to build our own database of polarized hydrogen profiles.

\subsection{Existing instruments}

We consider two existing interferometric instruments: the GI2T/REGAIN Interferometer (under commissioning in Southern France after a change of its visible detectors) and AMBER (under installation on the VLTI). Their characteristics are very complementary (Table 1). The combination of the
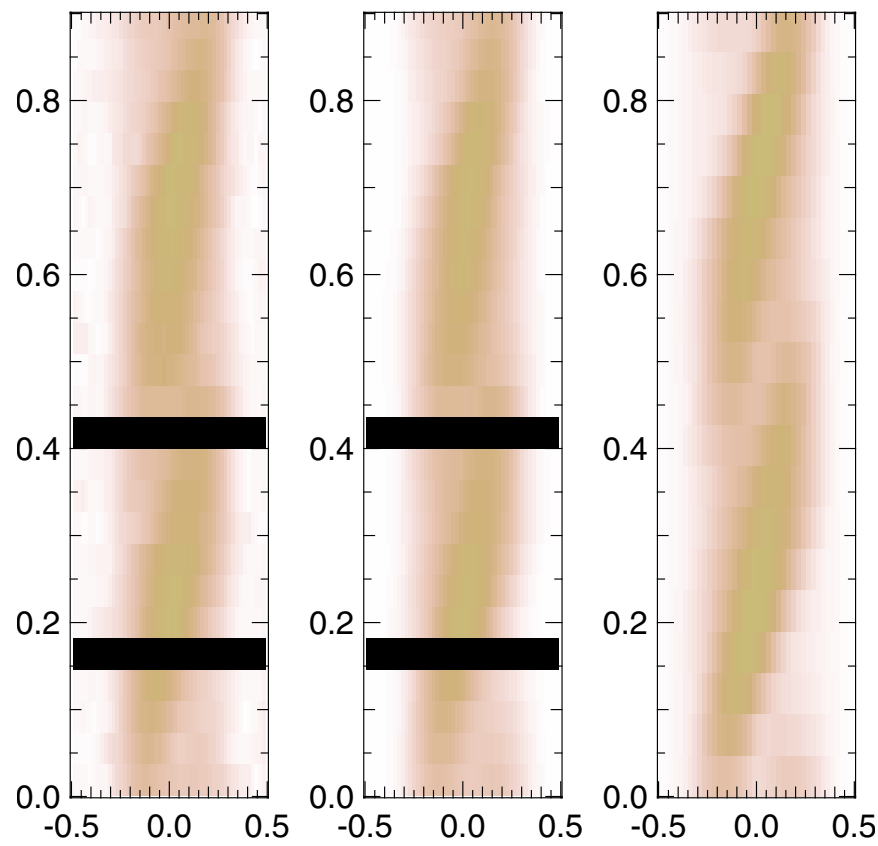

Fig. 5. Dynamical spectra of the Cr II 4824.13 lines observed (left) and computed (middle) by Kochukhov et al. (2002) and computed with our prloc code (right). Spectra around the stellar phases 0.15 and 0.4 have not been observed and thus simulated by Kochukhov et al. (2002).

two instruments gives access to information from the visible to the near-infrared range, which is very useful to probe different atmospheric layers of line-forming regions. Moreover the polarimetric mode of the GI2T (Chesneau et al. 2000) allows a magnetism study via differential fringe phase measurements in the $V$-Stokes parameter. The access of the near-infrared range with AMBER allows one to plan to study magnetism via resolved Zeeman patterns. The Zeeman effect increases quadratically with wavelength, while other line broadening effects (most particularly the Doppler effect) have a linear wavelength dependence: the magnetic field detection threshold and relative measurement uncertainties are accordingly lowered at longer wavelengths. As an example, AMBER has a spectral resolution of 10000 , i.e. $2 \AA$ at $\lambda=2 \mu \mathrm{m}$, while a line with a Landé factor of 1 and a magnetic field of $20 \mathrm{kG}$ has a Zeeman splitting of about $4 \AA$ at $2 \mu \mathrm{m}$.

As regards to instrumental resolution, we emphasize that:

- the spatial resolution $(\lambda / B)$ is similar for the GI2T in the visible and for AMBER in the $H$ band: improving the fringe phase signals proportional to $\boldsymbol{B} / \lambda$ (Eq. (2)) implies observing at the longest baseline and at the shortest wavelength (in the blue band for the GI2T and in the $J$ band for AMBER);

- the instrumental spectral channel width $\left(\Delta \lambda_{\text {instr }}=\lambda / \mathcal{R}\right.$ where $\mathcal{R}$ stands for the spectral resolution) of both instruments are similar;

- the intrinsic spectral resolution $\mathcal{R}$ has also to be considered. The Doppler half width $\Delta \lambda_{\text {Doppler }}$ is proportional to $\lambda v \sin i / c$ where $c$ denotes the speed of light and $v \sin i$ the target rotational velocity. Thus the sampling of a Doppler profile is directly linked to the intrinsic spectral resolution $\mathcal{R}$ : having $\Delta \lambda_{\text {instr }}=2 \Delta \lambda_{\text {Doppler }}$ requires $\mathcal{R}=c / 2 v \sin i$. The line sampling is three times better with the GI2T. 
Table 1. Characteristics of the GI2T/REGAIN Interferometer and the AMBER instrument of the VLTI.

\begin{tabular}{ccccccc}
\hline \hline Instruments & $\begin{array}{c}\text { Combined } \\
\text { telescopes }\end{array}$ & $\begin{array}{c}\text { Maximum } \\
\text { baseline }(\mathrm{m})\end{array}$ & $\begin{array}{c}\text { Spectral } \\
\text { range }\end{array}$ & $\begin{array}{c}\text { Spectral } \\
\text { resolution }\end{array}$ & $\begin{array}{c}\text { Polarimetric } \\
\text { mode }\end{array}$ & $\begin{array}{c}\text { Differential phase } \\
\text { accuracy }\end{array}$ \\
\hline GI2T & 2 & 65 & Visible & up to 30000 & Yes & $0.07 \mathrm{rd}(*)$ \\
AMBER & 3 & 200 & $J, H, K$ & up to 10000 & No & $10^{-3} \mathrm{rd}$ \\
\hline
\end{tabular}

(*) Measured by Vakili et al. (1998).

Finally, in terms of accuracy, the GI2T has allowed differential fringe phase measurements within an error bar of $0.07 \mathrm{rd}$ (Vakili et al. 1998) and an improvement of one magnitude seems to be achievable with the updated acquisition device. AMBER will ensure fringe phase measurements within a $10^{-3}$ rd error bar and aims at reaching $10^{-5}$ rd by means of dedicated calibration devices (Petrov et al. 2000).

\subsection{Abundance maps of $\alpha^{2} \mathrm{CVn}$}

We have studied the case of the CP star prototype, $\alpha^{2} \mathrm{CVn}$, for which some abundance maps already exist. We have assumed the magnetic field topology and abundance maps reconstructed by Kochukhov et al. (2002) from Magnetic Doppler Imaging. We have performed our simulation in the visible range $(\lambda=$ $4824 \AA$ ) with a baseline of $50 \mathrm{~m}$ for an angular diameter of 0.88 mas, a rotational speed $v \sin i$ of $15 \mathrm{~km} \mathrm{~s}^{-1}$ and a chromium abundance variation of 3 dex. We have calculated with the intensity code prloc (with $T_{\text {eff }}=11500 \mathrm{~K}$ and $\log g=4$ ) the fringe phases of several selected lines of various species $(\mathrm{Fe}$, $\mathrm{Cr}, \mathrm{Si}$ ) at a number of stellar rotational phases (see Fig. 7 for Cr II $\lambda 4824$ line). The $x$ and $y$ fringe phases $\left(\psi_{I}\right)$ follow the stellar spot location: at stellar phase $\phi=227^{\circ}$, we can locate a large overabundance at the top left and a smaller one at the bottom right. We obtain $y$ fringe phase effects larger than $15^{\circ}$ and $x$ fringe phase effects of a few degrees for an infinite spectral resolution. In terms of signal amplitude, this is detectable with the existing instruments.

In practice, the spectral resolution is limited and the phase signals are averaged over a strip of the spectral line. If the spectral line is not resolved (for a spectral resolution lower than about 10000 in the case of $\alpha^{2} \mathrm{CVn}$ ), the phase signals are integrated throughout the whole line and a part of the close continuum and thus can decrease by a factor 2 . Within this context (zone A in Fig. 6), the phase signal is proportional to the spectral resolution. From a spectral resolution of about 20000 , the stellar features through the spectral line are resolved (zone B in Fig. 6) and the phase signals are of the same order as with an infinite spectral resolution (zone C in Fig. 6). Given their respective accuracy and spectral resolution, AMBER and the GI2T appear as good candidates to detect such stellar spots.

\subsection{Magnetic field topology of $\beta \mathrm{CrB}$}

As a second test case, we have simulated the magnetic field topology of the well-known magnetic star, $\beta \mathrm{CrB}$. We have adopted the two magnetic field configurations described by

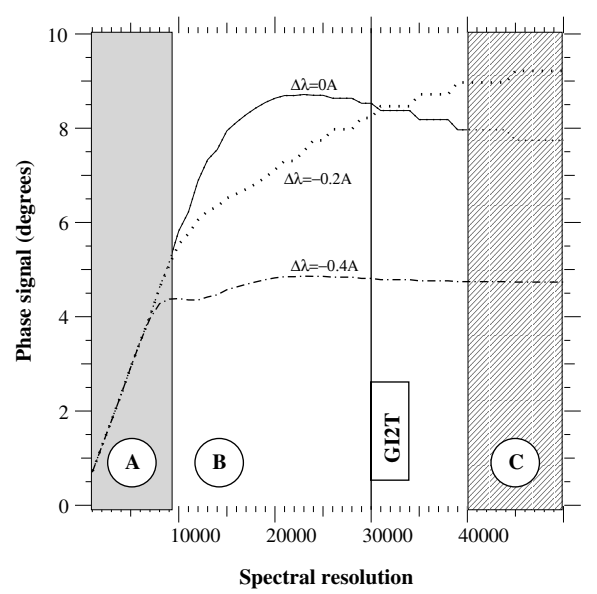

Fig. 6. Fringe phase signals (in degrees) vs. spectral resolution for a spectral channel centered at the central wavelength, $\lambda_{0}=4824 \AA$ (solid line), in the close blue wing (dotted line), and in the far blue wing (dash-dotted line). See text for comments about zones A, B and C.

Bagnulo et al. (2000), which are a superposition of a dipole and a non-linear quadrupole centered on the star. By inverting spectro-polarimetric data, they derived two possible models whose parameters are listed in Table 2 with the conventions of Fig. 8. We have also assumed a stellar angular diameter of 1 mas, a rotational velocity $v \sin i=3.5 \mathrm{~km} \mathrm{~s}^{-1}$ and a Voigt profile for the modeled lines. We have used the polarized radiative model (modpol) to compute the fringe phases $\psi_{V}$ of Fe I $\lambda 6430.8$ for the two magnetic models. We have plotted them as a function of the interferometric baseline orientation and versus the stellar phase (Fig. 9).

The two models are different in terms of interferometric signatures: the whole variation amplitude reaches $4.6^{\circ}$ for Model 1, while it reaches $2.6^{\circ}$ for Model 2 for a baseline of $50 \mathrm{~m}$ (GI2T/REGAIN Interferometer). Moreover, the phase variations are in antiphase for the two models: at a given baseline orientation or at a given stellar phase, the photocenter is not in the same hemisphere for the two models, as clearly shown in the reconstructed field maps displayed in Fig. 9 (right). The two models could therefore be disentangled provided that we sample adequately the stellar period and measure fringe phases smaller than $1^{\circ}$ with a $50-\mathrm{m}$ baseline, which can be attempted with the polarimetric mode of the GI2T and an accurate data processing. These fringe phase effects would be twice as large with a 100-m baseline. 


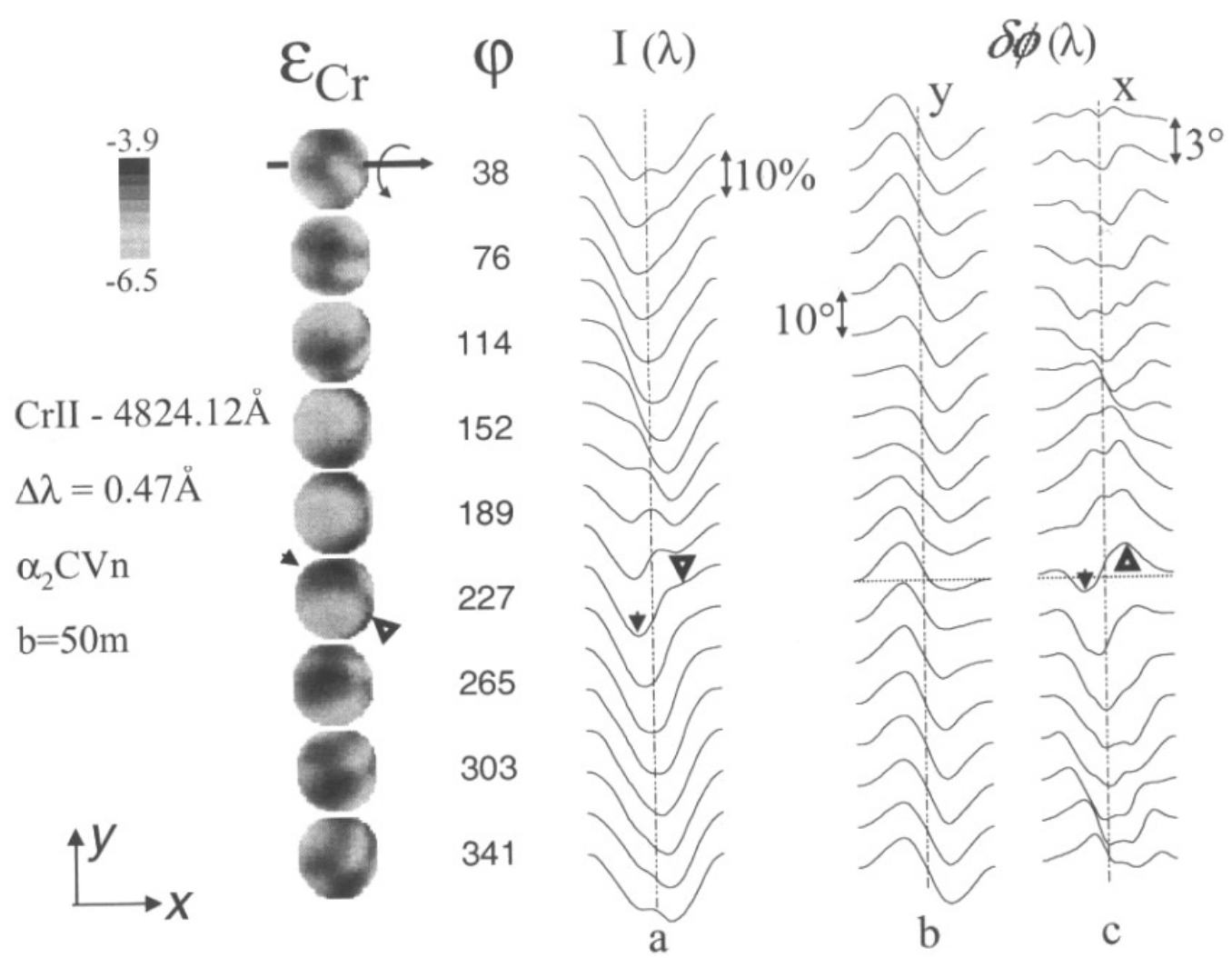

Fig. 7. Integrated spectra $I(\lambda)$ a) and photocenter $P(\lambda)$ in the $y$ b) and the $x$ c) directions of the Cr II $\lambda 4824$ line for a 50-m baseline and an infinite spectral resolution. The profiles extend over $\pm 0.47 \AA$ from the line center (dotted line). Spectra are vertically shifted by an amplitude factor of 0.2 , the $Y$ fringe phases are shifted by $-10^{\circ}$ and the $X$ fringe phases by $-3^{\circ}$ for consecutive rotational phases. Abundance maps at the left allow to follow the different spots over the stellar phase (overabundances are in dark colors as it is shown on the abundance scale at the top left).

Table 2. Magnetic topology of the models of $\beta \mathrm{CrB}$ derived by Bagnulo et al. (2000) from spectro-polarimetric data.

\begin{tabular}{llllllllll}
\hline \hline Model & $i\left(^{\circ}\right)$ & $\beta_{0}\left({ }^{\circ}\right)$ & $\beta_{1}\left({ }^{\circ}\right)$ & $\beta_{2}\left({ }^{\circ}\right)$ & $\gamma_{0}\left({ }^{\circ}\right)$ & $\gamma_{1}\left({ }^{\circ}\right)$ & $\gamma_{2}\left({ }^{\circ}\right)$ & $B_{d}(\mathrm{G})$ & $B_{q}(\mathrm{G})$ \\
\hline$\# 1$ & 168 & 88 & 12 & 79 & 124 & 350 & 331 & 12165 & 14429 \\
$\# 2$ & 163 & 93 & 40 & 114 & 35 & 143 & 35 & 8810 & 15145 \\
\hline
\end{tabular}

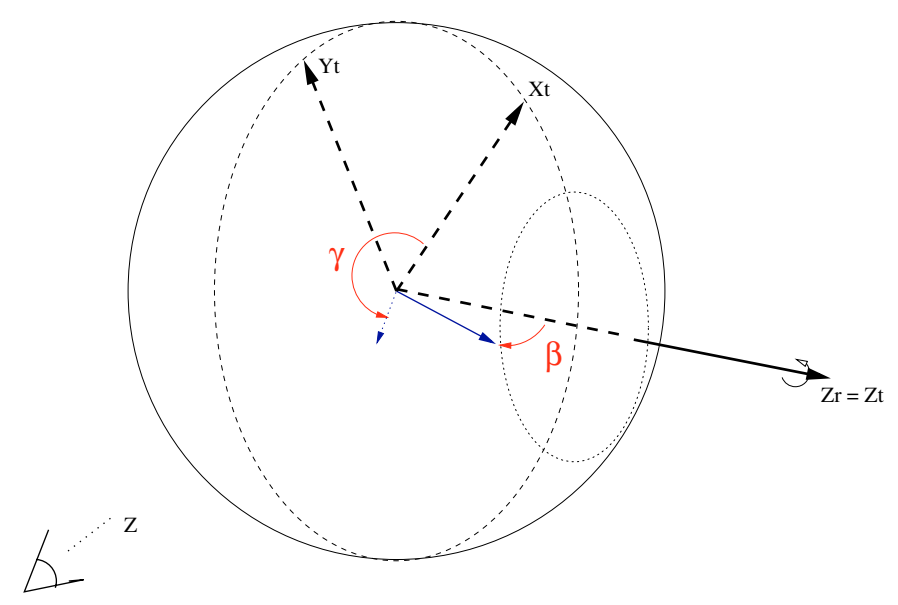

Fig. 8. Angle conventions for multipolar magnetic field topologies defined by Bagnulo et al. (2000). Each component of the magnetic field (dipolar and quadrupolar ones) are defined by two angles, $\beta$ and $\gamma$.

\section{Instrumental application}

\subsection{Instrumental and inversion requirements}

- Baseline length. Both kinds of science cases (magnetic field topology and abundance maps) will clearly take advantage of the long baseline in terms of amplitudes of the fringe phase signals: the longer the baseline, the higher the fringe phase effects and the less demanding the measurement accuracy requirement. For a given measurement accuracy, the maximal baseline defines the smallest star that could be observed and thus the extent of the scientific program that can be proposed. Baselines of a few hundred meters and even of one kilometer with OHANA will give access to the third moment of the flux distribution and provide subtle information on the features of the stellar surfaces (Lachaume 2003). In this context, the fringe phase signals are no longer proportional to the baseline length.

- Baseline orientation. Both applications will obviously benefit from a large number of telescopes: three simultaneous 

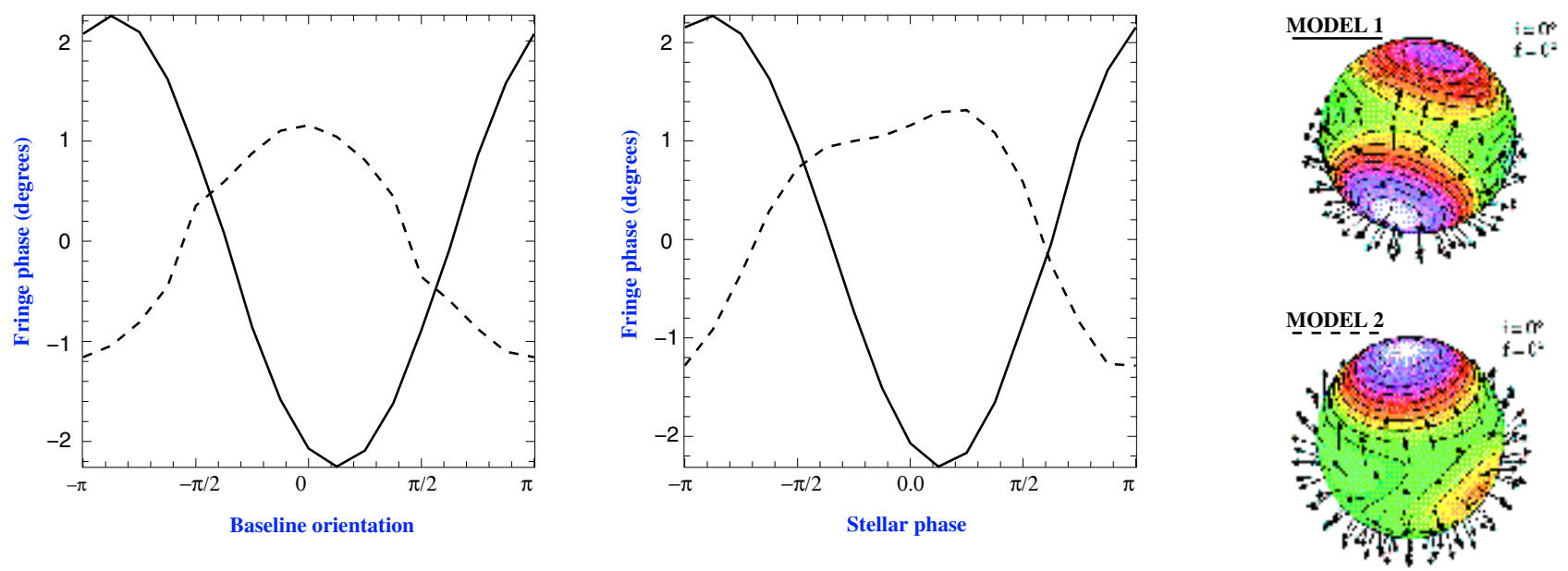

Fig. 9. Fringe phase in the $V$-Stokes parameter in the Fe I line $(\lambda=6430.844 \AA)$ versus the baseline orientation $(l e f t)$ and the stellar phase (middle) for the Model 1 (solid line) and the Model 2 (dashed line). The spectral resolution is 30000 and the fringe phase is computed in the spectral channel for which the phase effect is maximal. The baseline length is $50 \mathrm{~m}$. The two rightmost pictures (from Bagnulo et al. 2000) correspond to the field topology for Model 1 (top) and Model 2 (bottom) as seen from the North rotation pole $\left(i=0^{\circ}\right)$. The modulus of the magnetic field is visualized by means of different colors, with contour lines $1 \mathrm{kG}$ apart. The direction of the magnetic field is represented by unit vectors.

fringe phase measurements along different baseline orientations strongly constrain magnetic and abundance topologies. Moreover the more various the baseline orientations, the higher the probability to record a maximal fringe phase effects on one baseline since these effects are related to the angle between the star rotation axis on the sky and the baseline orientation. The first observations of $\mathrm{CP}$ stars with the three-beam AMBER instrument will be a promising step towards such imaging. These observations will also constitute new material to derive the imaging requirements within the field of CP stars of a future four-, six- or eight-telescope VLTI mode (Kern et al. 2003).

- Spectral Resolution. The observational strategy clearly depends upon this instrumental specification: the higher the spectral resolution, the finer the resolved structures. Nevertheless the ability to detect a fringe phase effects is not simply proportional to the spectral resolution as illustrated in the first science case. In fact, the spectral resolution has to be suited to the stellar features' size (Fig. 6).

- Measurement accuracy. For both applications (magnetic field topology and abundance maps), accurate fringe phase measurements at the degree level are required, which will be achievable with the currently planned instruments such as AMBER. Obviously, the expected fringe phase signals strongly depend on stellar characteristics that are in our science cases the chemical abundances and the magnetic field strength.

- Inversion algorithms. Two-dimensional maps require an inversion of interferometric data similar to Zeeman or Magnetic Doppler Imaging techniques. Adding the first moment information (i.e. the interferometric fringe phase information) in an existing inversion algorithm dedicated to spectral data has been performed and used to reconstruct non-radial pulsations (Jankov et al. 2001). Another application on abundance map reconstruction is in progress and comparisons between simulated maps of $\epsilon \mathrm{UMa}$ and those reconstructed by Doppler imaging by Rice \& Wehlau (1990) show that abundance maps of higher quality can be obtained by considering the interferometric information provided by the existing interferometers and their current noises (see the detailed reconstruction of $\epsilon \mathrm{UMa}(\Phi=$ 1.3 mas) in Jankov et al. (2003) thanks to a regularized inversion by the Maximum Entropy method). The next step is the reconstruction of magnetic maps, which is obviously more difficult because of the vector nature of the magnetic field.

\subsection{Observational strategies}

The previous section clearly shows that all the requirements are inter-dependant and that various observational strategies have to be defined according to the observing program or the available instruments.

First, coupling visible and infrared observations would be very interesting since they will provide new insight into the variation of the physical properties of the photosphere in its third (vertical) dimension. This approach is particularly relevant as observational evidence has been accumulated in recent years supporting the view that chemical abundances are vertically stratified in CP star atmospheres (Babel \& Lanz 1992) and that the pressure and temperature structure may depart considerably from standard models (Cowley et al. 2001).

Then, existing baselines and instruments already would allow us to constrain abundance maps of typical CP stars smaller than 1 mas. It is difficult to give the smallest stellar diameter our method will work with since the measured signals are strongly affected by the amplitude of abundance inhomogeneities and/or the magnetic field strength. Obviously longer baselines will allow us either to map smaller targets or to detect fringe phase effects on stars with fainter abundance inhomogeneities. Access to fainter abundance inhomogeneities is also possible via an improvement of the detection threshold of the interferometers. 
Table 3. Short-term observing program on the GI2T/REGAIN Interferometer and the AMBER instrument of the VLTI. For AMBER we mention the targets of our accepted guarantee time (GT) proposal.

\begin{tabular}{ccccccc}
\hline \hline Target & Sp. type & Magnitude & $\begin{array}{c}\text { Period } \\
(\text { days })\end{array}$ & $\begin{array}{c}v \sin i \\
\left(\mathrm{~km} \mathrm{~s}^{-1}\right)\end{array}$ & Application & Instrument \\
\hline$\epsilon \mathrm{UMa}$ & A0p & $V=1.8, J=1.72$ & 5.09 & 35 & Abundance/Magnetism & GI2T \\
$\alpha^{2} \mathrm{CVn}$ & A0p & $V=2.9, J=3.04$ & 5.47 & 15 & Abundance/Magnetism & GI2T \\
$\beta \mathrm{CrB}$ & A9p & $V=3.7, J=3.4$ & 18.5 & 3.5 & Magnetism & GI2T/AMBER \\
HD 128898 & A0p & $V=J=3.2$ & 4.48 & 15 & Abundance & AMBER (GT) \\
HD 203006 & A0p & $V=J=4.7$ & 2.12 & 50 & Abundance & AMBER (GT) \\
\hline
\end{tabular}

The full abilities of AMBER have to be investigated during the commissioning phase which will begin in 2004.

Finally, according to the target rotation velocity $v \sin i$ or to the available spectral sampling (linked to the spectral resolution $\mathcal{R}$ and to the pixel number in the line $n b_{\text {pixel }}$ ), we can distinguish various strategies:

- $n b_{\text {pixel }}=1$ (i.e. $v \sin i \geq 7.5 \mathrm{~km} \mathrm{~s}^{-1}$ with AMBER and $v \sin i \geq 2.5 \mathrm{~km} \mathrm{~s}^{-1}$ with the GI2T): we are only able to lift degeneracies of existing (Z)-DI maps by providing one point per stellar phase of differential fringe phase between a metallic line and the continuum;

- $n b_{\text {pixel }}=2$ (i.e. $v \sin i \geq 15 \mathrm{~km} \mathrm{~s}^{-1}$ with AMBER and $v \sin i \geq 5 \mathrm{~km} \mathrm{~s}^{-1}$ with the GI2T): we record, for each stellar phase, differential fringe phases in the blue and red wings of the metallic line under study. These measurements allow us to resolve the non-uniqueness of the (Z)-DI solutions. Moreover the rotation axis determination of stellar rotators is a by-product of such interferometric measurements and is a very useful input for the $(\mathrm{Z}) \mathrm{DI}$ data processing;

- $n b_{\text {pixel }}$ exceeds 7 or 8 (for $v \sin i \geq 60 \mathrm{~km} \mathrm{~s}^{-1}$ with AMBER and $v \sin i \geq 20 \mathrm{~km} \mathrm{~s}^{-1}$ with the GI2T): this provides various fringe phase measurements throughout the spectral line and allows us to refine reconstructed maps by providing an enhanced spatial information and by reinforcing the stellar limb areas. Within this instrumental context, an abundance map reconstruction of $\epsilon \mathrm{UMa}\left(v \sin i=35 \mathrm{~km} \mathrm{~s}^{-1}\right)$ has been simulated by Jankov et al. (2003).

As a consequence, astrophysical application will take advantage of coupling the very high spectral resolution observations of (Z)DI with the high angular resolution of interferometry.

\subsection{Short-term observing program}

Our short-term observing program (Table 3) aims at probing the feasibility on the GI2T and then using the ultimate performances of the GI2T and AMBER to improve the quality of $2 \mathrm{D}$ abundance and magnetic maps. For the preliminary program of the GI2T, we first identify:

- two CP stars $\left(\epsilon \mathrm{UMa}, \alpha^{2} \mathrm{CVn}\right)$ whose abundance maps are well known (e.g., Lueftinger et al. (2003) for $\epsilon \mathrm{UMa}$ and Kochukhov et al. (2002) for $\alpha^{2} \mathrm{CVn}$ ). They constitute ideal test targets for our inversion algorithm. Despite their small angular diameters (photometric estimations give $\Phi\left(\alpha^{2} \mathrm{CVn}\right)=0.8$ mas and $\Phi(\epsilon \mathrm{UMa})=1.4$ mas $)$ their strong abundance inhomogeneities lead us to expect large phase effects;

- $\beta \mathrm{CrB}$ for which two magnetic field topologies have been proposed by Bagnulo et al. (2000). It will allow us to test the performances of the polarimetric mode of the GI2T/REGAIN Interferometer in circularly polarized light. Note that $\beta \mathrm{CrB}$ could also be studied in infrared with AMBER.

Abundance observations with the GI2T/REGAIN are foreseen in 2004 as soon as the new visible detectors are commissioned whereas magnetic topology observations require the sky qualification (planned in 2004) of the polarimetric mode.

For the Guaranteed Time of AMBER two bright Ap stars have been selected, HD 128898 and HD 203006, whose periods of $4.48 \mathrm{~d}$. and $2.12 \mathrm{~d}$. respectively allow a correct sampling of the stellar phases. HD 128898 exhibits rotational modulation of line profiles (Kochukhov \& Ryabchikova 2001) which appears to be low because this star is seen close to the rotational pole $\left(i \sim 30^{\circ}\right)$. Because of this inclination, abundance inhomogeneities cannot be studied efficiently with standard spectroscopic methods like Doppler imaging. Our method will be perfectly suited to analyse this target.

This Guaranteed Time will occur after the Assembly, Integration and Verification period and the commissioning runs of AMBER planned throughout 2004. Further observations of $\mathrm{CP}$ stars can be proposed in open time after having deeply analyzed the data of the first observations in Guaranteed Time.

\section{Conclusion}

This paper shows how spectro-(polari)metric interferometry is a powerful tool to study stellar magnetism and activity. Provided that stars are partially resolved, differential fringe phases can bring constraints at the sub milliarcsecond scale on stellar rotator orientations on the sky, mass-loss phenomena, abundance inhomogeneities and magnetic topologies. Such a differential technique is required to resolve features on small targets like CP stars. In this paper, we report results of numerical simulations and propose observing programs on CP stars for the GI2T/REGAIN currently under commissioning after change of the detectors, and for the AMBER instrument under installation at the focus of the VLTI. For our application of mapping abundance inhomogeneities and magnetic fields, we show that signals expected on typical CP stars are detectable in the visible and in the infrared with both instruments. We emphasize that such interferometric information will allow us 
to significantly improve the quality of reconstructed images of stellar surfaces. We also emphasize that a detailed modelling of the observed objects is essential to correctly and fully interpret the future data to be provided by the optical aperture synthesis array and, even before that, to define the observational strategy and prepare the observations (selection of baselines, wavelengths, spectral lines, etc). Inversion algorithms also must be developed for an optimal use of the planned imaging arrays. The additional information recorded in polarized light will open new avenues to answer key questions on the role of magnetic fields in stellar physics. That is why the question of equipping a kilometric array such as OHANA and/or a second generation instrument of the VLTI with a polarimetric mode (Vakili et al. 2001) has to be studied as well as the suitable spectral resolution.

Acknowledgements. The authors want to warmly thank D. Mourard, F. Vakili and G. Mathys for their scientific support and fruitful discussions as well as C. Van't Veer for the spectra of CP stars. They thank the referees for their constructive comments that improved the quality of the paper. The authors are grateful to French Programs PNPS, PNST and CNRS/ATI for funding the SPIN project, and T. Lanz acknowledges the support of the Observatoire de Paris.

\section{References}

Babel, J., \& Lanz, T. 1992, A\&A, 263, 232

Bagnulo, S., Landolfi, M., Mathys, G., \& Landi Degl'Innocenti, M. 2000, A\&A, 358, 929

Bério, P., Stee, Ph., Vakili, F., et al. 1999, A\&A, 345, 203

Brillant, S., Lanz, T., Mathys, G., \& Stehlé, C. 1998, A\&A, 339, 286

Cassinelli, J. P., \& Hoffman, N. M. 1975, MNRAS, 173, 789

Chesneau, O., Rousselet-Perraut, K., Vakili, F., et al. 2000, in Interferometry in Optical Astronomy, SPIE Conf., ed. A. Quirrenbach, \& P. Léna, Proc. SPIE, 4006, 531

Chesneau, O., Vakili. F., Rousselet-Perraut, K., \& Stehlé, C. 2001, in Magnetic fields across the Hertzsprung-Russell diagram, ed. G. Mathys, S. K. Solanki, \& D. T. Wickramasinghe, ASP Conf., 248, 633

Chesneau, O., Wolf, S., \& Domiciano de Souza, A. 2003, A\&A, 410, 375

Colavita, M. 1992, in High-Resolution Imaging by Interferometry II, Ground-Based Interferometry at Visible and Infrared Wavelengths, ed. J. M. Beckers, \& F. Merkle, ESO Conf., 845

Cowley, C., Hubrig, S., Ryabchikova, T. A., et al. 2001, A\&A, 367, 939

Donati, J. F. 1996, in Stellar surface structure, IAU Conf., ed. G. Strassmeier, \& J. Linsky, 176, 53

Glindemann, A., Algomedo, J., Amestica, R., et al. 2003, in Interferometry for Optical Astronomy II, SPIE Conf., August 2002, Hawaii, 4838, 89

Hanbury Brown, R., Davis, J., \& Allen, L. R. 1974, MNRAS, 168, 93

Hubeny, I., \& Lanz, T. 2000, Am. Astron. Soc. Meet., 197, \#78.12

Jankov, S., Vakili, F., Domiciano de Souza, A., \& Janot-Pacheco, E. 2001, A\&A, 377, 721
Jankov, S., Domiciano de Souza, A., Stehlé, C., et al. 2003, in Interferometry for Optical Astronomy II, SPIE Conf., August 2002, Hawaii, 4838, 587

Jennison, R. C. 1958, MNRAS, 118, 276

Kern, P., Malbet, F., Berger, J. P., et al. 2003, in Interferometry for Optical Astronomy II, SPIE Conf., August 2002, Hawaii, 4838, 312

Kochukhov, O., \& Ryabchikova, T. 2001, A\&A, 377, L22

Kochukhov, O., Piskunov, N., Ilyin, I., et al. 2002, A\&A, 389, 420

Kurucz, R. L. 1993, VizieR On-line Data Catalog: VI/39

Lachaume, R. 2003, A\&A, 400, 795

Lagarde, S., Sanchez, L., Petrov, R. 1995, in Tridimensional Optical Spectroscopic Methods in Astrophysics, ed. G. Comte, \& M. Marcelin, ASP Conf. Ser., 71, 360

Landstreet, J. 1980, AJ, 85, 611

Leblanc, F., Michaud, G., \& Babel, J. 1994, ApJ, 431, 388

Leroy, J. L., Landolfi, M., \& Landi Degl'Innocenti, E. 1993, A\&A, 270,335

Lueftinger, T., Kuschnig, R., Piskunov, N., \& Weiss, W. 2003, A\&A, 406, 1033

Mathys, G. 1988, A\&A, 189, 179

Mathys, G., Stehlé, C., Brillant, S., \& Lanz, T. 2000, A\&A, 358, 1151

Michaud, G. 1970, ApJ, 160, 641

Moss, D. 2001, in Magnetic fields across the Hertzsprung-Russell diagram, ed. G. Mathys, S. K. Solanki, \& D. T. Wickramasinghe, ASP Conf., 248, 305

Mourard, D., Bonneau, D., Stee, P., et al. 2002, in Interferometry for Optical Astronomy II, SPIE Conf., August, Hawaii, 4838, in press

Perrin, G., Lai, O., Woillez, J., et al. 2003, in Interferometry for Optical Astronomy II, SPIE Conf., August 2002, Hawaii, 4838, 1290

Petrov, R. 1988, in High-Resolution Imaging by Interferometry, ESO Conf., March, ed. F. Merkle, Garching, Germany, 235

Petrov, R., Malbet, F., Weigelt, G., et al. 2000, in Interferometry in Optical Astronomy, SPIE Conf., ed. A. Quirrenbach, \& P. Léna, Proc. SPIE, 4006, 68

Piskunov, N., \& Kochukhov, O. 2002, A\&A, 381, 736

Rice, J. B., \& Wehlau, W. H. 1990, A\&A, 233, 503

Rousselet-Perraut, K., Vakili, F., \& Mourard, D. 1996, Opt. Eng., 35, 2943

Rousselet-Perraut, K., Vakili, F., Mourard, D., et al. 1997, A\&AS, 123, 173

Rousselet-Perraut, K., Chesneau, O., Berio, P., \& Vakili, F. 2000, A\&A, 354, 595

Ryabchikova, T., Piskunov, N., Kochukhov, O., et al. 2002, A\&A, 384, 545

Rybicki, G. B., \& Hummer, D. G. 1991, A\&A, 245, 171

Semel, M. 1989, A\&A, 225, 456

Stehlé, C., Brillant, S., \& Mathys, G. 2000, Eur. Phys. J. D, 11, 491

Vakili, F. 1981, A\&A, 101, 352

Vakili, F., Mourard, D., Bonneau, D., et al. 1997, A\&A, 323, 183

Vakili, F., Mourard, D., Stee, Ph., et al. 1998, A\&A, 335, 261

Vakili, F., Chesneau, O., Delplancke, F., et al. 2001, in Interferometry for Optical Astronomy II, ESO Workshop Proc., ed. G. Monnet, \& J. Bergeron, June, Garching

Wittkowski, M., Schöller, M., Hubrig, S., et al. 2002, Astron. Nachr., 323,241 